\section{Stellungnahme der Landesärztekammer Hessen und der Bundesärztekammer}

Mit der Frage des Mehrfachansatzes der Ziffer 263 GOÄ je Kalendertag bei Durchführung einer spezifischen Immuntherapie (Hyposensibilisierung) hat sich die Bundesärztekammer bereits 2003 befasst, mit folgendem Ergebnis: Die Hyposensibilisierungsbehandlung wird eingeleitet mit einer Dosissteigerungsphase, in deren Verlauf in üblicherweise wöchentlichen Intervallen Allergenextrakte in aufsteigender Dosierung subkutan injiziert werden. Durchschnittlich erstreckt sich diese Behandlungsphase bis zum Erreichen der tolerablen Maximaldosis über ca. 12 Wochen, danach folgt die Dosiserhaltungsphase.

Falls aus medizinischen Gründen ein besonders schneller Therapieerfolg erzielt werden soll (vorzugsweise bei Sensibilisierung gegen Insektengift, gelegentlich auch bei Inhalationsallergenen), kann eine sogenannte Schnellhyposensibilisierung (RushVerfahren) durchgeführt werden. Beim konventionellen Rush-Verfahren wird nach ca. fünf Tagen die Maximaldosis erreicht. Erforderlich sind etwa vier Injektionen täglich mit aufsteigenden Allergenkonzentrationen in einem Intervall von drei bis vier Stunden. Bei dem sogenannten Ultra-Rush-Verfahren, das in der Regel unter stationären Bedingungen durchgeführt wird, kann dieses Ziel in zwei bis drei Tagen erreicht werden. Verabreicht werden hier fünf bis sechs Injektionen pro Tag mit aufsteigenden Allergenkonzentrationen in etwa zweistündigen Abständen.

Außer bei den genannten Schnellhyposensibilisierungsbehandlungen ist unter Umständen auch in den Fällen, bei denen mehrere Sensibilisierungen (z. B. gegen saisonale Allergene) vorliegen, die Injektion von zwei verschiedenen Allergenextrakten in getrennten Injektionsvorgängen an einem Tag erforderlich.

Im Anschluss an jede Injektion wird der Patient wegen der Gefahr allergischer Allgemeinreaktionen mindestens 30 Minuten im Arbeitsbereich des Arztes nachbeobachtet. Der Leistungslegende der Ziffer 263 GOÄ zufolge ist die Hyposensibilisierungsbehandlung je Sitzung einmal berechnungsfähig. Obligat beinhaltet ist eine Nachbeobachtungszeit. Deren Dauer ist in der Leistungslegende zwar nicht ausdrücklich angegeben, sollte jedoch mindestens 30 Minuten betragen, da eine eventuelle allergische Allgemeinreaktion innerhalb dieses Zeitraums auftritt.

Diese Beobachtungsdauer wird nach den Leitlinien der Deutschen Gesellschaft für Allergologie und klinische Immunologie und des Ärzteverbands Deutscher Allergologen zur praktischen Durchführung der spezifischen Immuntherapie mit Allergenen empfohlen.

Für die Definition des Merkmals „Sitzung“ können zwei Sozialgerichtsurteile herangezogen werden: So heißt es in einem Urteil des Bundessozialgerichts vom 24. August 1994 (AZ 6 RKa 40/92), dass eine Sitzung den gesamten Zeitraum umfasst, in dem sich der Patient in der Praxis eines Arztes zur Untersuchung und/oder Behandlung befindet. Eine Sitzung umfasst auch mehrere Zeitintervalle, wenn der Patient zwischendurch aus medizinischen oder praxisorganisatorischen Gründen oder wegen seines Zustands unbehandelt warten muss oder wenn er zeitweise allein durch das Praxispersonal behandelt wird.

Nach einem Urteil des Landessozialgerichts Stuttgart vom 4. September 1996 (AZ L 5 Ka 2851/95) liegt eine neue Sitzung dann vor, wenn eine Leistung nach ihrer eigenen Typik in mehrere Phasen aufgeteilt werden muss und diese sich nicht unmittelbar aneinander anschließen können.

Auch wenn zu konzedieren ist, dass diese Auslegungen aus den zitierten Sozialgerichtsurteilen nicht ohne weiteres auf die GOÄ übertragbar sind, ist aus Sicht der Bundesärztekammer in besonders gelagerten Fällen wie z. B. bei dem Einsatz des RushVerfahrens oder bei Verwendung von zwei unterschiedlichen Allergenextrakten die Ziffer 263 GOÄ im Verlauf eines Tages bei einem Patienten mehrfach ansetzbar.

Voraussetzung ist dabei, dass nach Abschluss einer Phase der Hyposensibilisierungsbehandlung - also Injektion einer bestimmten Allergenkonzentration oder eines bestimmten Allergenextrakts mit anschließender obligatorischer Nachbeobachtungszeit sich eine oder mehrere neue Phasen - also Injektion einer anderen Allergenkonzentration oder eines anderen Allergenextrakts einschließlich obligater Nachbeobachtung anschließen, ein mehrfacher Ansatz entsprechend der durchgeführten Phasen ist dann gerechtfertigt. Für die Transparenz der Abrechnung empfiehlt die Bundesärztekammer, die erneute Berechnung unter Angabe der Uhrzeit vorzunehmen.

Brief zum Mehrfachansatz der Ziffer 263 GOÄ vom 30. Juni 2010
Bundesoberbehörde (Paul-Ehrlich-Institut) überprüft werden.

Der Gesetzgeber hat in der TAV vorgeschrieben, dass für jegliche Therapieallergene, die mit einem oder mehreren TAV-Allergenen gemischt werden (also zum Beispiel auch Kräuterpollen, Schimmelpilze oder Tierepithelien), ein zulassungspflichtiger Mischextrakt entsteht. Seitdem diese gesetzlichen Vorgaben bestehen, sind mehr als $90 \%$ aller Mischungen von Therapieallergenen vom Markt verschwunden. Somit sind die behandelnden Allergologen heute gezwungen, bei polysensibilisierten $\mathrm{Pa}$ tienten mit mehreren Therapie-Extrakten zu behandeln - früher verwendete Mischungen sind schlichtweg nicht mehr erhältlich.

\section{Der Begriff der Therapiesitzung}

In einem Urteil des Bundessozialgerichts vom 24. August 1994 (AZ 6 RKa 40/92) wird eine Sitzung als der gesamte Zeitraum gefasst, in dem sich der Patient in der Praxis eines Arztes zur Untersuchung und/oder Behandlung befindet.

Nach einem Urteil des Landessozialgerichts Stuttgart vom 4. September 1996 (AZ L 5 Ka 2851/95) liegt eine neue Sitzung dann vor, wenn eine Leistung nach ihrer eigenen Typik in mehrere Phasen aufgeteilt werden muss und diese sich nicht unmittelbar aneinander anschließen können.

Aus Sicht der Bundesärztekammer ist in besonders gelagerten Fällen die Ziffer 263 GOÄ im Verlauf eines Tages bei einem Patienten mehrfach ansetzbar - z. B. beim Einsatz besonderer SIT-Verfahren wie dem Rush-Verfahren oder bei Verwendung von unterschiedlichen Allergenextrakten [Klakow-Franck R. GOÄRatgeber „Je Sitzung“. Deutsches Ärzteblatt 2003; 100 (30): A-20302003].

Aus allergologisch-fachlichen Aspekten existiert daher kein Zweifel an der Möglichkeit eines Mehrfachansatzes der Ziffer 263 bei mehreren Therapiesitzungen an einem Tag. Es ist für die Allergologen sehr erfreulich, dass die Bundesärztekammer bereits 2003 eine klare Stellungnahme zugunsten der Abrechenbarkeit einer modernen Immuntherapie abgegeben hat, die von der Landesärztekammer Hessen bestätigt wurde.

Referat Sozial- und Berufsrecht des AeDA 